\title{
Do Feeding and Eating Disorders Fit the General Definition of Mental Disorder?
}

\author{
M. Cristina Amoretti ${ }^{1}$ (D)
}

Published online: 25 August 2020

(c) The Author(s) 2020

\begin{abstract}
This paper aims at considering the conceptual status of feeding and eating disorders (FEDs). Now that the Diagnostic and Statistical Manual of Mental Disorders (DSM-5) has changed the classification and some relevant criteria of FEDs, it is particularly relevant to evaluate their psychiatric framework and their status as mental disorders. I focus my efforts on addressing only one specific question: Do FEDs fit the DSM-5 general definition of mental disorder? In DSM-5 a mental disorder is defined as a syndrome that reflects a dysfunction and is usually associated with significant distress or disability. More importantly, there is an explicit statement saying that all mental disorders listed in the manual must meet the requirements highlighted by the general definition. Thus, I evaluate whether or not FEDs are really meant to reflect a dysfunction and are usually associated with significant distress or disability.
\end{abstract}

Keywords Disability $\cdot$ Distress $\cdot$ Dysfunction $\cdot$ DSM-5 $\cdot$ Feeding and eating disorders (FEDs) $\cdot$ Mental disorder

\section{Introduction}

This paper aims at considering the conceptual status of feeding and eating disorders (FEDs). Now that the Fifth Edition of the Diagnostic and Statistical Manual of Mental Disorders (DSM-5) has changed the overall classification and some specific criteria of FEDs, it is important to evaluate their psychiatric frameworks and their status as mental disorders. I focus my efforts in this analysis on clarifying one conceptual dimension of the overall FEDs debate by addressing only one specific question: Do FEDs fit the DSM-5 general definition of mental disorder? Or, to put it differently, are FEDs mental disorders in line with the DSM-5's criteria? In DSM-5, a mental disorder is defined as a syndrome that reflects a dysfunction and is usually associated with significant distress or disability (American Psychiatric Association 2013a); thus, a mental disorder seems to be identified with a harmful dysfunction (Wakefield 1992). In DSM-5 there is also an explicit statement saying that all mental disorders listed in the manual must meet the requirements highlighted

M. Cristina Amoretti

cristina.amoretti@unige.it

1 DAFIST, Philosophy Section \& PhilHeaD, Research Center for Philosophy of Health and Disease, University of Genoa, Via Balbi 4, 16126 Genova, Italy by their general definition (American Psychiatric Association 2013a, 20). Thus, I evaluate whether or not FEDs can reasonably be said to reflect a dysfunction and whether they are usually associated with distress or disability. The question of whether or not FEDs can be regarded as mental disorders is conceptually interesting and important in its own right and it has practical and ethical consequences, too. However, in the present context, I will not focus on these consequences.

The paper is structured as follows. First, I briefly discuss the nosological history and the most relevant features of FEDs as well as the main requirements of the DSM-5 general definition of mental disorder. Then, I stress the importance of identifying the specific dysfunctions that underlie FEDs, as the definition of mental disorder demands, since the presence of certain symptoms alone is not sufficient to indicate a mental disorder. Next, I focus on the harm requirement, trying to unpack the two notions of distress and disability in the specific case of FEDs and to understand their role as diagnostic criteria. Finally, I conclude with some considerations about the nature of FEDs. 


\section{FEDs in DSM-5}

In DSM-5, FEDs are "characterized by a persistent disturbance of eating or eating-related behavior that results in the altered consumption or absorption of food and that significantly impairs physical health or psychosocial functioning" (American Psychiatric Association 2013a, 329). They are all included in a standalone chapter which comprises six independent disorders and two residual categories ${ }^{1}$ : pica, rumination disorder ( $\mathrm{RD})$, avoidant/restrictive food intake disorder (ARFID), anorexia nervosa (AN), bulimia nervosa (BN), binge eating disorder (BED), other specified feeding or eating disorder (OSFED), and unspecified feeding or eating disorder (UFED). The diagnostic criteria for RD, ARFID, AN, BN, and BED are construed as "mutually exclusive, so that during a single episode, only one of these diagnoses can be assigned" (American Psychiatric Association 2013a, 329).

The new classification has some important differences from the one included in DSM-IV-TR (Attia et al. 2013). First, the diagnostic criteria for $\mathrm{AN}$ and $\mathrm{BN}$ have been refined and expanded to decrease the number of people diagnosed in the DSM-IV-TR residual category of eating disorders not otherwise specified (EDNOS). ${ }^{2}$ The DSM-5 criteria thus include in the main diagnoses of $\mathrm{AN}$ and $\mathrm{BN}$ many patients previously classified as EDNOS (Caudle et al. 2015).

Second, DSM-IV-TR categories of pica, RD, and feeding disorder of infancy or early childhood-previously included in the chapter describing disorders usually first diagnosed in infancy, childhood, or adolescence-were incorporated among FEDs. The latter category, which was rarely used, has been renamed as ARFID and the diagnostic criteria have been expanded to include individuals of any age. Small changes were also made to the criteria for pica and $\mathrm{RD}$, for clarification and for extending them to individuals of any age.

Third, BED—which in DSM-IV-TR was included in the Appendix, among conditions needing further studies-was officially recognized among FEDs as an independent category. As for BN, the required minimum average frequency

\footnotetext{
${ }^{1}$ Even if DSM-5 maintains a categorical approach to diagnosis and classification, it also introduces dimensional aspects via specific diagnoses (American Psychiatric Association 2013b). This is the case, for example, of autism spectrum disorder (ASD) and substance use disorder, that merge four and two categorical diagnoses into a single spectrum, respectively.

2 This was a major shortcoming of DSM-IV-TR: for instance, as a 2012 study reported (Le Grange et al. 2012), in the US $80.97 \%$ of adolescents and $75.38 \%$ of adults diagnosed with an eating disorder were classified as having EDNOS, which, however, was of less clinical utility.
}

of binge eating was reduced from twice to once a week over the last 3 months.

The decision to unite pica, RD, and ARFID with AN, $\mathrm{BN}$, and BED in a single chapter named "Feeding and Eating Disorders" suggests that some of these conditions are feeding disorders (FDs), while some others are eating disorders (EDs). Nevertheless, in DSM-5 there is no explanation on which mental disorders belong to which group, nor an explicit definition of each group. The National Institute of Mental Health $(2018,2)$ defines EDs as "serious medical illnesses marked by severe disturbances to a person's eating behaviors. Obsessions with food, body weight, and shape may be signs of an eating disorder," but no equivalent definition for FDs is proposed. In the pediatric framework, FDs are regarded as "severe disruptions in nutritional and caloric intake exceeding ordinary variations in hunger, food preference, and/or interest in eating" (Sharp et al. 2017, 116); recently, a consensus definition has been proposed for (pediatric) FDs, which are defined "as impaired oral intake that is not age-appropriate, and is associated with medical, nutritional, feeding skill, and/or psychosocial dysfunction" (Goday et al. 2019, 124). The main difference between the two groups of disorders is that body-image disturbance and related thoughts and behaviors, such as fear of gaining weight or being fat, negative body talk, frequent bodychecking, or weighing, only characterize EDs; moreover, the presence of compensatory behaviors, such as self-induced vomiting, laxative abuse, fasting, or excessive exercise, is also only found in EDs. In the present context, I take pica, $\mathrm{RD}$, and ARFID to be FDs, ${ }^{3}$ and AN, BN, and BED to be EDs.

\section{The Definition of Mental Disorder in DSM-5}

Since the DSM-III (American Psychiatric Association 1980), the introduction of the manual has included an explicit definition of the general concept of mental disorder. This definition has slightly changed across the various editions of the manual (Amoretti and Lalumera 2018; Cooper 2015), but the main requirements are still the same, that is, dysfunction and harm (distress or disability):

A mental disorder is a syndrome $[\ldots]$ that reflects a dysfunction in the psychological, biological, or developmental processes underlying mental functioning. Mental disorders are usually associated with significant distress or disability in social, occupational, or

\footnotetext{
${ }^{3}$ Some scholars, however, have some doubts about considering ARFID a feeding disorder (Kennedy et al. 2018).
} 
other important activities (American Psychiatric Association 2013a, 20).

According to this definition, each mental disorder is an entity that features a list of specific symptoms and meets two different requirements. The first requirement is dysfunction, which is a necessary one; this means that no condition can be recognized as a mental disorder if it does not reflect an underlying dysfunction. The second requirement is twofold, as it involves distress or disability; in this case, a condition could in principle be recognized as a mental disorder even if it is not associated with neither distress nor disability, as they have to be usually (not necessarily) present.

With the DSM-5, an important statement has been added in the Introduction, explicitly saying that all mental disorders must meet the two requirements above (American Psychiatric Association 2013a, 20). This means that, besides meeting the various criteria listed in DSM- 5 for distinguishing one mental disorder from another, a certain condition can legitimately count as pathological, that is, as a mental disorder only if it: (1) reflects an underlying dysfunction, and (2) usually produces some distress or disability. To put it differently, specific syndromes do not stand alone with respect to their status as mental disorders because the general definition of mental disorder is their ultimate validator (Amoretti and Lalumera 2019a).

To start with the dysfunction requirement, it is important to distinguish between the underlying dysfunction, that is, the pathology, and the distal causes, that is, the etiology, of a certain condition. Of course, it is widely recognized that mental disorders in general, and FEDs in particular, are complex or multifactorial disorders, which have a heterogeneous etiology encompassing genetic, neurological, psychological, environmental, and sociocultural factors. Nevertheless, what is called for by the general definition of mental disorder is the explication of the underlying pathological dysfunction, not of the whole etiology of the condition.

The main problem of the dysfunction requirement is that our current knowledge of the neurological and cognitive mechanisms underlying mental disorders is still vague and thus it is difficult to indicate what specific dysfunction is reflected by a certain syndrome. For this reason, First and Wakefield (2013) have argued that the symptoms listed in the diagnostic criteria of mental disorders must be pathosuggestive, that is, they must clearly point to an underlying dysfunction, as in the case of delusions or hallucinations. Most symptoms, however, are not pathosuggestive per se, as they describe mere behaviors, which as such may be intentional actions or normal reactions to external stressors. When symptoms are not pathosuggestive per se, First and Wakefield suggest considering them with some specifications and/or together with additional diagnostic criteria, to secure the connection to an underlying dysfunction. These specifications and additional diagnostic criteria can regard the duration and persistence of symptoms, their frequency and intensity, their disproportionality with respect to an environmental trigger, their pervasiveness in various contextual settings, as well as contextual exclusion for scenarios where symptoms are better understood as normal cultural practices or normal reactions to external stressors. Moreover, a minimum number of symptoms that must be simultaneously present in a syndrome can also be indicated to increase pathosuggestivity.

Even admitting that syndromes can be accommodated to become sufficiently pathosuggestive, another problem arises. As Horwitz puts it, "Using the criterion of dysfunction to define mental disorders implies that the presence of symptoms alone is never sufficient to indicate a mental disorder" (Horwitz 2002, 22). In other words, for the dysfunction to play the epistemic or individuating role demanded by the general definition of mental disorder, it must be explained and operationalized. If the presence of a dysfunction remains a mere working hypothesis, then the dysfunction requirement would be nothing more than an idle conceptual wheel (Amoretti and Lalumera 2019a). ${ }^{4}$

Moving to the harm requirement, it can be implemented among the diagnostic criteria with more ease. It would be sufficient to add a specific criterion explicitly demanding a significant level of distress and disability (First and Wakefield 2013), as it is done for many mental disorders listed in DSM-5. Still, there is a problem with determining what distress and disability mean and what their role is as diagnostic criteria (Amoretti and Lalumera 2019b).

In DSM-5 there is no explicit definition of distress, but in the literature, distress has been equated to a state of depression or anxiety (Wheaton 2007), or a state of worry, concern, or suffering (Ustün and Kennedy 2009); still, there can be a wide range of interpretations of the diagnostic criteria in which distress is mentioned. Moreover, DSM-5 does not provide any clear explanation of how to discriminate between diagnostically relevant and irrelevant distress (Phillips 2009). As for disability, in DSM-5 it is equated with functional impairment, which refers to the limitations in the social, occupational, and other important areas of everyday life that are due to a mental disorder (Ustün and Kennedy 2009). The International Classification of Functioning, Disability and Health (ICF) defines disability as an umbrella term for impairments, activity limitations, and participation

\footnotetext{
${ }^{4}$ DSM, since DSM-III, is meant to be atheoretical, and thus to avoid any reference to the underlying dysfunctions of mental disorders. However, the general definition of mental disorder, which is the ultimate validator for distinguishing between disorders and normal conditions, explicitly requires the presence of a dysfunction. Thus, for the manual to be conceptually coherent, dysfunctions must be explained and operationalized.
} 
restrictions. Terminological differences aside, disability is generally considered a relational concept involving environmental and social components.

Among the diagnostic criteria of mental disorders, distress and disability can play at least three different roles (Amoretti and Lalumera 2019b). They can be used: (1) to make up for the current absence of relevant biomarkers or clinically useful measurements of severity, and thus to solve the threshold problem and distinguish between mental disorder and non-disorder, or between different mental disorders (this may be the case of anxiety disorders or neurocognitive disorders: these syndromes doubtless reflect a dysfunction, but it is unclear how one might assess their severity and establish the thresholds for demarcating normal and pathological anxieties, or mild and major neurocognitive disorders $)^{5}$; (2) to make up for the current lack of knowledge about the underlying dysfunction, and, again, to separate mental disorders from non-disorders (where the presence of a dysfunction is dubious, as with some paraphilic disorders, the harm criterion can provisionally be used to separate normal from pathological conditions); (3) to discriminate between those mental disorders that must be diagnosed and/ or medically treated and those mental disorders that should not (where the presence of a dysfunction is quite clear, as in the case of erectile disorder, the label of mental disorder should be applied; however, there might be good reasons not to diagnose or treat some of these conditions in practice, precisely when they are harmless).

In what follows, I apply the above considerations to the nosological categories of FEDs. First, I assess whether the symptoms of FEDs are sufficiently pathosuggestive and, if so, whether the underlying dysfunction plays the epistemic or individuating role required by the general definition. Second, I evaluate the presence of distress and disability, their specific meaning, as well as their possible role among the diagnostic criteria of FEDs.

\section{The Dysfunction Requirement and FDs}

The main diagnostic criterion for pica is "Persistent eating of non-nutritive, non-food substances over a period of at least 1 month" (American Psychiatric Association 2013a, 329). Of course, one cannot distinguish normality from pathology based on this symptom alone and with no precise reference to the context. The other three criteria are in fact contextual qualifiers aimed at excluding situations where the symptoms can be understood as normal developmental steps, culturally supported or socially normative practices,

\footnotetext{
5 This role is explicitly mentioned in the introduction of DSM-5 (American Psychiatric Association 2013a, 21).
}

or consequences of other pathological conditions. However, it is dubious whether the four criteria taken together are sufficiently pathosuggestive. To some scholars, pica may be even adaptive (Young 2011) and thus functional, at least for some theories of function. Of course, the persistent eating of non-nutritive, non-food substances is non-normal, but is it evidence of a specific underlying dysfunction?

Research has shown that pica is often associated with other medical conditions, among which insufficient diet and nutrient deficiency (particularly iron), brain lesions, diseases or infections of the gastrointestinal tract, pregnancy, as well as other mental disorders, such as intellectual disability, ASD, schizophrenia, and obsessive-compulsive and related disorders (Hartmann et al. 2012). This may suggest that the underlying dysfunction can vary from case to case. For instance, some scholars propose a gastrointestinal dysfunction, since by consuming substances such as earth, one would be able to reduce the bioavailability of pathogens and toxins in the gastrointestinal tract (Young et al. 2010), or a temporal lobe dysfunction, since certain brain lesions influence abnormal eating behaviors (Funayama 2017). As a consequence, pica might be better understood as a mere symptom (that is, a research entity, like fever or pain), rather than as an independent mental disorder (that is, a diagnostic entity), as it is currently presented in DSM-5. Indeed, historically pica has been long regarded as a symptom of other pathological conditions (Parry-Jones and Parry-Jones 1992).

A similar analysis can be made for $\mathrm{RD}$, for which the main diagnostic criterion is "Repeated regurgitation of food over a period of at least 1 month. Regurgitated food may be re-chewed, re-swallowed, or spit out" (American Psychiatric Association 2013a, 332). Even admitting that the whole syndrome is sufficiently pathosuggestive, it is not clear whether it corresponds to a single, specific underlying dysfunction.

Moving to ARFID, according to DSM-5, it is mainly characterized by:

apparent lack of interest in eating or food, [and/or] avoidance based on the sensory characteristics of food, [and/or] concern about/fear of aversive consequences of eating, as manifested by persistent failure to meet appropriate nutritional and/or energy needs associated with one (or more) of the following:

1 significant weight loss;

2 significant nutritional deficiency;

3 dependence on enteral feeding or oral nutritional supplements;

4 marked interference with psychosocial functioning (American Psychiatric Association 2013a, 334).

As in the previous cases, these symptoms alone are not sufficiently pathosuggestive, and thus further contextual 
qualifiers are indicated to exclude situations where the symptoms relate to food scarcity, cultural practices, or other pathological conditions (such as AN). Still, it is dubious whether the four criteria of ARFID are jointly sufficient to unambiguously indicate an underlying dysfunction. Assuming instead that the symptoms are sufficiently pathosuggestive, what kind of dysfunction can underlie them? DSM-5 only excludes a body-image disturbance (which, as we shall see in the next section, would point to AN).

ARFID involves a highly heterogeneous symptomatology where the common denominator is detrimental food restriction due to causes other than a body-image dysfunction. To make up for such heterogeneity, it has been proposed to introduce a subtype differentiation (Sharp and Stubbs 2019). Of course, a heterogeneous symptomatology that lacks specificity may simply reflect different ways in which a single underlying dysfunction is expressed. At present, there is a paucity of research into ARFID, due to its relatively recent inclusion in DSM-5 (Strand et al. 2019). However, ARFID can be present alongside other mental disorders such as ASD, ADHD, intellectual disability, and reactive attachment disorder. It has also been suggested that gastrointestinal and neurological dysfunctions, food allergies as well as hypersensitivity to taste or texture can be relevant to ARFID (Coglan and Otasowie 2019). In the current state of knowledge, it would be hard to theorize that the symptoms of ARFID depend on a single underlying dysfunction.

It is worth noting that, in the specific case of FDs, the exclusion criterion for other mental disorders is slightly different from that of most other DSM-5's mental disorders. The exclusion criterion, in fact, states that when the feeding behavior is sufficiently severe to deserve more clinical attention than usual, it can still give rise to a pica, RD, or ARFID diagnosis, even if such behavior clearly occurs in the context of another mental disorder. This suggests that a FDs diagnosis can be made regardless of the underlying dysfunction and thus strengthens the sense that FDs are not underpinned by specific dysfunctions. As a consequence, FDs could be better seen as research entities, rather than genuine nosological categories, which instead should meet the requirements of the general definition of mental disorder.

The symptoms of FDs do not seem to be sufficiently pathosuggestive or at least they do not point to characteristic underlying dysfunctions. Moreover, even admitting the presence of a specific underlying dysfunction, which would hold all the symptoms together, it would not play any substantive role in identifying pica, RD, and ARFID, and in demarcating them (as mental disorders) from similar behavioral patterns that are not mental disorders. The contextual qualifiers try to do this task instead, claiming that the relevant feeding behavior might be better understood in the light of normal developmental steps, food scarcity, cultural practices, or other pathological conditions.

\section{The Dysfunction Requirement and EDs}

The DSM-5 diagnostic criteria for AN are:

A. Restriction of energy intake relative to requirements, leading to a significantly low body weight in the context of age, sex, developmental trajectory, and physical health [...].

B. Intense fear of gaining weight or of becoming fat, or persistent behavior that interferes with weight gain, even though at a significantly low weight.

C. Disturbance in the way in which one's body weight or shape is experienced, undue influence of body weight or shape on self-evaluation, or persistent lack of recognition of the seriousness of the current low body weight (American Psychiatric Association 2013a, 338-339).

Even if the main feature of $\mathrm{AN}$ is a consistent reduction of food intake leading to a significant weight loss (Giordano 2005, 22), criterion A per se does not point to any dysfunction, nor it is inherently pathosuggestive. Moreover, it is not sufficient to distinguish AN from ARFID, or from similar behavioral patterns that are not mental disorders, such as hunger strikes. The addition of criterion $B$ does not change the situation because the intense fear of gaining weight or becoming fat-which may be specific for AN and perhaps considered pathosuggestive-is just mentioned as a disjunct and is thus not necessary for a diagnosis of AN (a mere behavior that interferes with weight gain would be in itself sufficient). However, the dysfunction requirement seems to be explicitly called for by criterion $\mathrm{C}$, which is constituted by three disjuncts.

First, a "disturbance in the way in which one's body weight or shape is experienced", mainly exhibited by the fact that patients with AN overestimate their body shape and weight (Molbert et al. 2017), may point to an underlying body-image or body-experience/perception dysfunction; some recent findings suggest, for instance, a disturbance of long term body representation (Gadsby 2017), a locked allocentric representation of one's body (Riva 2012, 2014; Riva and Gaudio 2018), a poor or diminished interoceptive awareness (Herbert and Pollatos 2012; Khalsa et al. 2015; Merwin et al. 2010), or a deficit in the integration of multisensory bodily signals and representations (Case et al. 2012; Zopf et al. 2016). From a phenomenological perspective, the presence of a disturbance of embodiment has been proposed, that is, an anomalous contrast between lived body (Leib) and physical body (Koerper), or between body-subject and body-object (Kuhle 2019).

Second, an "undue influence of body weight or shape on self-evaluation," may point to the presence of 
a dysfunction of the system for evaluating self-worth: whereas most subjects evaluate themselves based on their perceived performances in different spheres of life, such as work, family, friends, or sports, subjects with AN evaluate themselves mostly or exclusively based on their weight and shape, and their ability to control them, especially through their eating habits (Castellini et al. 2014; Fairburn et al. 2003). Recent literature proposes, for instance, the presence of a disturbance of self-identity, produced by the interconnection between the disturbance of the experience of one's own body and the process of shaping one's own personal identity (Stanghellini et al. 2015).

Third, a "persistent lack of recognition of the seriousness of the current low body weight," mainly exhibited by the denial of one's own illness (Vandereycken 2006a), may also point to the presence of a dysfunction (Vandereycken 2006b), such as an impaired interoceptive awareness (Papezova et al. 2005), a dysfunction in information processing (Casper 1998), a lack of insight (Konstantakopoulos et al. 2011), or an inability to effectively regulate emotions (Haynos and Fruzzetti 2011).

Overall, the symptoms of AN, specifically criterion C, are pathosuggestive and clearly point to a dysfunction. This means that AN meets the dysfunction requirement of the DSM-5's general definition of mental disorder. However, at least some of the hypothesized dysfunctions of AN are thought to be in common with $\mathrm{BN}$ and, more importantly, to some mental disorders other than EDs. For instance, the body-image dysfunction seems also to underlie muscle dysmorphia (MD), which in DSM-5 is listed as a subtype of body dysphoric disorder and is regarded as a condition in which the principal symptom is a marked preoccupation with one's body being insufficiently muscular-despite often being of above-average muscularity and lean body mass (Blomeley et al. 2018). This means that the underlying dysfunction plays only partially an epistemic or individuating role in identifying and demarcating AN from other mental disorders. More precisely, the presence of a body image dysfunction may determine an EDs diagnosis, as opposed to a FDs diagnosis, but it is unhelpful to discriminate $\mathrm{AN}$ from its neighboring disorders, such as $\mathrm{BN}$ or $\mathrm{MD}$.

Moving to $\mathrm{BN}$, its diagnostic criteria are:

A. Recurrent episodes of binge eating. An episode of binge eating is characterized by both of the following:

1 Eating, in a discrete period of time [...], an amount of food that is definitely larger than what most individuals would eat in a similar period of time under similar circumstances.

2 A sense of lack of control over eating during the episode $[\ldots]$.
B. Recurrent inappropriate compensatory behaviors in order to prevent weight gain, such as self-induced vomiting; misuse of laxatives, diuretics, or other medications; fasting; or excessive exercise.

$\mathrm{C}$. The binge eating and inappropriate compensatory behaviors both occur, on average, at least once a week for three months.

D. Self-evaluation is unduly influenced by body shape and weight.

E. The disturbance does not occur exclusively during episodes of anorexia nervosa (American Psychiatric Association 2013a, 345).

Criteria A and C are not sufficiently pathosuggestive, as they can easily include anyone who binges on food once a week for 3 months, a behavior which may be unhealthy but not dysfunctional-criterion $\mathrm{C}$ has been weakened in comparison with DSM-IV-TR, resulting in a higher rate of BN diagnoses (Ornstein et al. 2013). Moreover, defining what is "an amount of food definitely larger than what most individuals would eat" is difficult to evaluate, especially considering that most fast-foods and many other restaurants serve meals that are two to three times the size of a recommended meal. The addition of criterion B is still not enough to make the syndrome pathosuggestive: even if recurrent inappropriate compensatory behaviors aimed at preventing weight gain is judged not to be normal, this is not enough to consider it dysfunctional. Putting aside criterion E, which is an exclusionary criterion for $\mathrm{AN}$, let us focus on criterion $\mathrm{D}$, which is equivalent to the second disjunct of criterion $\mathrm{C}$ for $\mathrm{AN}$.

That self-evaluation is unduly influenced by body shape and weight - which seems to be particularly critical in $\mathrm{BN}$ (Blechert et al. 2011) - may point to a dysfunction of the system for evaluating self-worth and be explained by a disturbance of self-identity. Given that the underlying dysfunction seems to be the same of $\mathrm{AN}$, and that is not uncommon to encounter individuals who initially met the diagnostic criteria for $\mathrm{AN}$, then those of $\mathrm{BN}$ and now have a mixed state (Fairburn and Cooper 2011), some scholars have questioned the presence of two distinct nosological entities and proposed to consider EDs as a spectrum, also including some OS/UFEDs (Brooks et al. 2012). ${ }^{6}$ Should the underlying dysfunction be the same, considering a unique nosological category or a spectrum with a heterogeneous symptomatology would be more in line with the general definition of mental disorder. In the current state of affairs, however, the underlying dysfunction plays only partially an epistemic or

\footnotetext{
${ }^{6}$ As I previously mentioned, DSM-5 still retains a categorical approach, despite the presence of some sparse dimensional categories. This means that introducing a spectrum in the case of AN and $\mathrm{BN}$ (with the possible inclusion of some OS/UFEDs) may clash with the overall categorical approach of DSM-5.
} 
individuating role in identifying and demarcating $\mathrm{BN}$ from other mental disorders (as in the case of AN).

Considering BED, its diagnostic criteria resemble the part of $\mathrm{BN}$ in which the subject overindulges on food (criterion A) but lack the part in which he or she adopts recurrent compensatory behaviors to prevent weight gain. Moreover:

B. The binge-eating episodes are associated with three (or more) of the following:

1 Eating much more rapidly than normal.

2 Eating until feeling uncomfortably full.

3 Eating large amounts of food when not feeling physically hungry.

4 Eating alone because of feeling embarrassed by how much one is eating.

5 Feeling disgusted with oneself, depressed, or very guilty afterward.

C. Marked distress regarding binge eating is present.

D. The binge eating occurs, on average, at least once a week for three months.

E. The binge eating is not associated with the recurrent use of inappropriate compensatory behavior as in bulimia nervosa and does not occur exclusively during the course of bulimia nervosa or anorexia nervosa (American Psychiatric Association 2013a, $350)$.

As in the case of BN, criteria A and D (which in DSM-5 reduced the binge eating from twice to once a week) are not sufficiently pathosuggestive. Moreover, putting aside criterion $\mathrm{E}$, which is an exclusionary criterion for $\mathrm{AN}$ and $\mathrm{BN}$, the addition of other criteria does not yet seem sufficient to clearly indicate a dysfunction. First, criterion B simply describes five kinds of behaviors that, despite being not normal, do not point to a dysfunction. Criterion $\mathrm{C}$, in turn, makes the presence of harm, in the form of distress, necessary. However, it is at least controversial that being distressed regarding binge eating would be enough to point to an underlying dysfunction. As Wakefield points out "Feelings of being out of control and of self-disgust are understandable as internalized social value judgments about such behavior rather than as evidence of the presence of genuine compulsive pathology" (Wakefield 2016, 122).

Even considering the BED syndrome as a whole, it seems unsuited for indicating a specific dysfunction. According to Wakefield, for example, BED is unable to pinpoint any real dysfunctional behaviors: "It remains unclear why the common tendency to overeat when food is plentifully available (especially when food is presented to tempt us) is classifiable as a disorder versus a normal variation of an evolutionarily shaped inclination to amass scarce calories while one can (even though this natural inclination may be problematic in our food-rich environment)" (Wakefield 2016, 122).

Introducing BED as a new mental disorder in DSM-5 has been widely criticized, indeed (Frances and Nardo 2013; Wakefield 2013, 2016), as this diagnosis covers too much of normal life-so that it has also jokingly been called a bar mitzvah and wedding disorder (Wakefield 2013) —and turns the vice of gluttony into a disorder (Frances 2012). In general, the research on BED seems to indicate that this diagnosis is rather different from $\mathrm{AN}$ and $\mathrm{BN}$, as it does not underlie a dysfunction of body-image/experience, selfidentity, and so on. For what we have seen above, it is dubious that it underlies any dysfunction at all. If no dysfunction is clearly indicated by the symptoms, we can conclude that it does not meet the general definition of mental disorder.

In conclusion, it is worth mentioning OS/U diagnoses, which in DSM-5 indicate genuine, independent nosological categories and still account for a substantial percentage of all FEDs diagnoses. In the case of FEDs, OSFED includes five specific subcategories for conditions that either do not meet the full criteria for any of the FEDs, as purging disorder and night eating syndrome, or represent subthreshold conditions, as atypical AN, as well as BN and BED of low frequency and/or limited duration. The situation is similar for UFED diagnoses: they do not meet the full criteria for any of the FEDs but, in this case, the reasons are not made explicit. In all the above cases, there is no reference to a dysfunction, either explicitly or implicitly, through a list of pathosuggestive symptoms. For this reason, Wakefield (2016) maintains that all DSM-5 OS/U diagnoses should be revised to require that the symptoms are likely better explained by an underlying dysfunction than by a normal variation, thus satisfying the dysfunction requirement. At present, OSFED and UFED represent a contradiction in DSM-5, as they do not meet the dysfunction requirement. ${ }^{7}$

\section{Harm Requirement and FEDs}

According to the DSM-5, the harm requirement - that is, the presence of distress or disability—is not necessary for being a mental disorder. Still, it is important to understand if and how such a requirement is used in FEDs diagnoses.

Let us start with FDs. Among the diagnostic criteria of pica there is no explicit harm criterion; nonetheless, pica is considered one of the more dangerous of self-harming

\footnotetext{
${ }^{7}$ It is worth noting that introducing a spectrum in the case of AN and $\mathrm{BN}$ may allow one not to regard OS/UFEDs as a contradiction in the DSM-5 anymore. In fact, as some OS/UFEDs can be included in the spectrum, they might be considered clinically significant atrisk behaviors or sub-threshold conditions. I thank an anonymous reviewer for pointing to this theoretical implication.
} 
behaviors and can easily result in premature death. Even if distress and disability can be absent, this condition represents an important risk factor for many pathologies, such as poisoning, gastrointestinal obstruction, and so on. Similarly, $\mathrm{RD}$ and ARFID do not contain any specific harm requirement; while the latter represents an important risk factor for malnutrition and premature death, the former probably does not. That being said, it is important to clarify that talking about risk factors and harm is very different. The concept of risk factor comes from epidemiology, where it is regarded as a variable associated with an increased risk of disease and has nothing to do with distress or disability as characterized above.

Moving to EDs, there is no explicit harm criterion in the diagnostic criteria of $\mathrm{AN}$ and $\mathrm{BN}$. In the case of AN, the "intense fear of gaining weight or becoming fat" can represent a manifestation of distress, but it is just a disjunct of criterion B, so it may well be absent. Anxiety, and thus distress in one of its meanings, is often associated with AN and $\mathrm{BN}$, but, again, this is not made explicit in the diagnostic criteria of these two conditions. According to DSM-5, individuals with $\mathrm{AN}$ and $\mathrm{BN}$ may exhibit a range of functional limitations associated with the disorder: social isolation and/ or failure to fulfill academic or career potential or severe impairment in the social-life domain (American Psychiatric Association 2013a, 343 and 349); these specifications, however, are not included among diagnostic criteria. Thus, the presence of harm is not necessary for a diagnosis of AN and BN. Still, they are life-threatening conditions, often associated with serious physical deterioration and death.

The case of BED is different as it includes an explicit harm criterion: "Marked distressed regarding binge eating is present" (American Psychiatric Association 2013a, 350). In this case, it is reasonable to suspect that this criterion has been made explicit to make up for the current lack of knowledge about the underlying dysfunction and pathosuggestivity of the whole syndrome, and, thus, to separate pathological from normal conditions. As Wakefield pointed out, however, it is at least controversial that being distressed regarding binge eating would be sufficient to indicate the presence of a genuine pathology and make the binge eating a mental disorder.

To recap, excluding the case of BED, the harm requirement plays no specific role in FEDs. In principle, most FEDs can be diagnosed even if the disordered subject does not experience any distress or disability. On the one hand, this possibility does not contradict the DSM-5 general definition of mental disorder, as the harm requirement is not necessary for being a mental disorder. On the other hand, that FEDs can be in principle diagnosed without the presence of distress or disability is important because the disordered subject might not be the best person to judge and evaluate his or her own harm. As we have seen above, many people with FEDs do not recognize that they have a pathology and deny the dangerousness of their condition. At one extreme, "pro-ana" (pro AN) and "pro-mia" (pro BN) movements explicitly claim that $\mathrm{AN}$ and $\mathrm{BN}$ are not mental disorders but rather sustainable lifestyle choices; in their view, AN and $\mathrm{BN}$ eating behaviors are indeed an exception from the norm, but they are neither dysfunctional nor harmful (Boero and Pascoe 2012). ${ }^{8}$ If the harm criterion would be enlisted among the diagnostic criteria of FEDs, then those who do not experience any distress or disability could not be diagnosed with a mental disorder and thus properly treated.

\section{Conclusions}

In this paper, I tried to assess whether FEDs fit the DSM-5 general definition of mental disorder. In so doing, I focused my attention especially on the dysfunction requirement, as it is the necessary one, and analyzed the harm requirement only briefly.

First, I tried to evaluate to what extent the diagnostic criteria of FEDs are sufficiently pathosuggestive.

In the case of FDs, the pathosuggestivity of symptoms, as described in DSM-5, is at least questionable, especially because they do not point to any specific dysfunction but seem instead to be compatible with the presence of different kinds of underlying dysfunctions. Moreover, the dysfunction requirement does not seem to play any epistemic or individuating role in FDs. If so, it is possible to conclude that FDs do not meet the dysfunction requirement. Of course, this does not mean that FDs are useless constructs, as they are able to single out and recognize relevant groups of patients, but that they would be probably better conceived as research entities, like fever or pain, rather than as independent nosological categories. The cases of AN and BN are different, as their symptoms seem to be sufficiently pathosuggestive. Even if the research is not conclusive, and both conditions remain somehow elusive, there is a general agreement that they reflect some kind of body-image or body-experience dysfunction, and thus meet the dysfunction requirement. Still, the underlying dysfunction is not fully operationalized and

\footnotetext{
${ }^{8}$ Someone may suggest that pro-ana and pro-mia movements could be framed in more positive terms, as this is increasingly happening with the Mad Pride and neurodiversity movements. However, it is important to stress that $\mathrm{AN}$ and $\mathrm{BN}$, if compared to other mental disorders, are potentially life-threatening conditions that can have serious medical consequences for an individual's health. As for AN, there are, for instance, an increasing risk for heart failure (due to abnormally slow heart rate and low blood pressure), osteoporosis, muscle loss, kidney failure (as a result of dehydration), and chronic fatigue. As for $\mathrm{BN}$, there are an increasing risk for heart failure (due to the electrolyte imbalance caused by purging behaviors), gastric or esophageal rupture, peptic ulcers, and pancreatitis.
} 
plays only partially an epistemic or individuating role: it does differentiate EDs from FDs and non-disorders, but it does not differentiate $\mathrm{AN}$ from $\mathrm{BN}$ and other neighboring disorders. The possibility of introducing a unique nosological category $(\mathrm{AN}+\mathrm{BN})$ or a spectrum with a heterogeneous symptomatology would be thus more in line with the general definition of mental disorder. Finally, at present, BED and OS/UFEDs seem to contradict the general definition of mental disorder.

Moving to the harm requirement, I claimed that distress and disability do not play any substantial role among the diagnostic criteria of FEDs. The only exception is the case of BED where an explicit harm criterion has been included to make up for the current lack of knowledge about the underlying dysfunction and pathosuggestivity of the whole syndrome. This means that most FEDs can be diagnosed even if the subject does not experience any distress or disability. This does not contradict the DSM-5's general definition of mental disorder and allows a diagnosis even when the subject experiences no harm and does not recognize the dangerousness of his or her condition.

Assessing whether FEDs fit the DSM-5 general definition of mental disorder is important for both practical and theoretical reasons: demanding that each condition listed in DSM-5 meets the general definition of mental disorder not only prevents the diagnosis of false positives (Wakefield 1997, 2016) but is also necessary to ensure the coherence of the manual (Amoretti and Lalumera 2019a).

Acknowledgements Open access funding provided by Università degli Studi di Genova within the CRUI-CARE Agreement.

\section{Compliance with Ethical Standards}

Conflict of interest Author declares that she has no conflict of interest.

Ethical Approval This article does not contain any studies with human participants or animals performed by any of the authors.

Open Access This article is licensed under a Creative Commons Attribution 4.0 International License, which permits use, sharing, adaptation, distribution and reproduction in any medium or format, as long as you give appropriate credit to the original author(s) and the source, provide a link to the Creative Commons licence, and indicate if changes were made. The images or other third party material in this article are included in the article's Creative Commons licence, unless indicated otherwise in a credit line to the material. If material is not included in the article's Creative Commons licence and your intended use is not permitted by statutory regulation or exceeds the permitted use, you will need to obtain permission directly from the copyright holder. To view a copy of this licence, visit http://creativecommons.org/licenses/by/4.0/.

\section{References}

American Psychiatric Association (1980) Diagnostic and statistical manual of mental disorders, DSM-III, 3rd edn. American Psychiatric Association, Washington, DC

American Psychiatric Association (2013a) Diagnostic and statistical manual of mental disorders, DSM-5, 5th edn. American Psychiatric Publishing, Washington, DC

American Psychiatric Association (2013b) DSM-5's integrated approach to diagnosis and classification. American Psychiatric Publishing, Washington, DC. https://www.psychiatry.org/ File\%2520Library/Psychiatrists/Practice/DSM/APA_DSM5-Integrated-Approach.pdf. Accessed 1 July 2020

Amoretti MC, Lalumera E (2018) Il criterio del "danno" nella definizione di disturbo mentale del DSM. Alcune riflessioni epistemologiche. RIFP Rivista Internazionale di Filosofia e Psicologia 9(2):139-150

Amoretti MC, Lalumera E (2019a) A potential tension in DSM-5: the general definition of mental disorder versus some specific diagnostic criteria. J Med Philos 44(1):85-108

Amoretti MC, Lalumera E (2019b) Harm should not be a necessary criterion for mental disorder: some reflections on the DSM-5 definition of mental disorder. Theor Med Bioeth 40:321-337

Attia E et al (2013) Feeding and eating disorders in DSM-5. Am J Psychiatry 170(11):1237-1239

Blechert J, Ansorge U, Beckmann S, Tuschen-Caffier B (2011) The undue influence of shape and weight on self-evaluation in anorexia nervosa, bulimia nervosa and restrained eaters: a combined ERP and behavioral study. Psychol Med 41(1):185-194

Blomeley D, Phillipou A, Castle DJ (2018) Sizing it up: a systematic review of the nosology of muscle dysmorphia. Clin Res Psychol 1(1): $1-10$

Boero N, Pascoe CJ (2012) Pro-anorexia communities and online interaction: bringing the pro-ana body online. Body Soc 18(2):27-57

Brooks SJ, Rask-Andersen M, Benedict C, Schiöth HB (2012) A debate on current eating disorder diagnoses in light of neurobiological findings: is it time for a spectrum model? BMC Psychiatry 12:1-11

Case LK, Wilson RC, Ramachandran VS (2012) Diminished sizeweight illusion in anorexia nervosa: evidence for visuo-proprioceptive integration deficit. Exp Brain Res 217(1):79-87

Casper RC (1998) Behavioral activation and lack of concern, core symptoms of anorexia nervosa? Int J Eat Disord 24(4):381-393

Castellini G, Trisolini F, Ricca V (2014) Psychopathology of eating disorders. J Psychopathol 20:461-470

Caudle H, Pang C, Mancuso S, Castle D, Newton R (2015) A retrospective study of the impact of DSM-5 on the diagnosis of eating disorders in Victoria, Australia. J Eat Disord 3:35-39

Coglan L, Otasowie J (2019) Avoidant/restrictive food intake disorder: what do we know so far? BJPsych Adv 25(2):90-98

Cooper RV (2015) Must disorders cause harm? The changing stance of the DSM. In: Demazeux S, Singy P (eds) The DSM-5 in perspective: philosophical reflections on the psychiatric Babel. Springer, Dordrecht, pp 83-96

Fairburn CG, Cooper Z (2011) Eating disorders, DSM-5 and clinical reality. Br J Psychiatry 198(1):8-10

Fairburn CG, Cooper Z, Shafran R (2003) Cognitive behaviour therapy for eating disorders: a "transdiagnostic" theory and treatment. Behav Res Ther 41(5):509-528

First M, Wakefield JC (2013) Diagnostic criteria as dysfunction indicators: bridging the chasm between the definition of mental disorder and diagnostic criteria for specific disorders. Can J Psychiatry 58(12):663-669 
Frances AJ (2012) DSM-5 is a guide, not a bible: simply ignore its 10 worst changes. HuffPost. https://www.huffingtonpost.com/allen -frances/dsm-5_b_2227626.html. Accessed 1 July 2020

Frances AJ, Nardo JM (2013) ICD-11 should not repeat the mistakes made by DSM-5. Br J Psychiatry 203(1):1-2

Funayama M (2017) Pica after acquired brain injury and in degenerative diseases is associated with temporal lobe dysfunction and its related semantic memory deficits. J Alzheimer's Dis Parkinsonism 7(5):367-369

Gadsby S (2017) Distorted body representations in anorexia nervosa. Conscious Cogn 51:17-33

Giordano S (2005) Understanding eating disorders: conceptual and ethical issues in the treatment of anorexia and bulimia nervosa. Clarendon Press, Oxford

Goday PS et al (2019) Pediatric feeding disorder: consensus definition and conceptual framework. J Pediatr Gastroenterol Nutr 68(1):124-129

Hartmann AS, Becker AE, Hampton C, Bryant-Waugh R (2012) Pica and rumination disorder in DSM-5. Psychiatr Ann 42(11):426-430

Haynos A, Fruzzetti A (2011) Anorexia nervosa as a disorder of emotion dysregulation: theory, evidence, and treatment implications. Clin Psychol Sci Pract 18(3):183-202

Herbert BM, Pollatos O (2012) The body in the mind: on the relationship between interoception and embodiment. Top Cogn Sci 4(4):692-704

Horwitz AV (2002) Creating mental illness. University of Chicago Press, Chicago

Kennedy GA, Wick MR, Keel PK (2018) Eating disorders in children: is avoidant-restrictive food intake disorder a feeding disorder or an eating disorder and what are the implications for treatment? F1000Research 7(F1000 Faculty Rev):88-94

Khalsa SS, Craske MG, Li W, Vangala S, Strober M, Feusner JD (2015) Altered interoceptive awareness in anorexia nervosa: effects of meal anticipation, consumption and bodily arousal. Int J Eat Disord 48(7):889-897

Konstantakopoulos G, Tchanturia K, Surguladze SA, David AS (2011) Insight in eating disorders: clinical and cognitive correlates. Psychol Med 41(9): 1951-1961

Kuhle L (2019) Anorexia nervosa and the embodied mind. In: Tekin S, Bluhm R (eds) The Bloomsbury Companion to philosophy of psychiatry. Bloomsbury, London, pp 113-128

Le Grange D, Swanson SA, Crow SJ, Merikangas KR (2012) Eating disorder not otherwise specified presentation in the US population. Int J Eat Disord 45(5):711-718

Merwin RM, Zucker NL, Lacy JL, Elliott CA (2010) Interoceptive awareness in eating disorders: distinguishing lack of clarity from non-acceptance of internal experience. Cogn Emot 24(5):892-902

Molbert SC et al (2017) Depictive and metric body size estimation in anorexia nervosa and bulimia nervosa: a systematic review and meta-analysis. Clin Psychol Rev 57:21-31

National Institute of Mental Health (2018) Eating disorders: about more than food. NIH Publication, Bethesda, MD

Ornstein RM et al (2013) Distribution of eating disorders in children and adolescents using the proposed DSM-5 criteria for feeding and eating disorders. J Adolesc Health 53(2):303-305

Papezova H, Yamamotova A, Uher R (2005) Elevated pain threshold in eating disorders: physiological and psychological factors. J Psychiatr Res 39(4):431-438

Parry-Jones B, Parry-Jones WL (1992) Pica: symptom or eating disorder? A historical assessment. Br J Psychiatry 160(3):341-354
Phillips MR (2009) Is distress a symptom of mental disorders, a marker of impairment, both or neither? World Psychiatry 8(2):91-92

Riva G (2012) Neuroscience and eating disorders: the allocentric lock hypothesis. Med Hypotheses 78(2):254-257

Riva G (2014) Out of my real body: cognitive neuroscience meets eating disorders. Front Hum Neurosci 8(236):1-20

Riva G, Gaudio S (2018) Locked to a wrong body: eating disorders as the outcome of a primary disturbance in multisensory body integration. Conscious Cogn 59:57-59

Sharp WG, Stubbs KH (2019) Avoidant/restrictive food intake disorder: a diagnosis at the intersection of feeding and eating disorders necessitating subtype differentiation. Int $\mathbf{J}$ Eat Disord 52(4):398-401

Sharp WG, Volkert VM, Scahill L, McCracken CE, McElhanon B (2017) A systematic review and meta-analysis of intensive multidisciplinary intervention for pediatric feeding disorders: how standard is the standard of care? J Pediatr 181:116-124

Stanghellini G, Trisolini F, Castellini G, Ambrosini A, Faravelli C, Ricca V (2015) Is feeling extraneous from one's own body a core vulnerability feature in eating disorders? Psychopathology 48(1):18-24

Strand M, von Hausswolff-Juhlin Y, Welch E (2019) A systematic scoping review of diagnostic validity in avoidant/restrictive food intake disorder. Int J Eat Disord 52(4):331-360

Ustün B, Kennedy C (2009) What is 'functional impairment'? Disentangling disability from clinical significance. World Psychiatry $8(2): 82-85$

Vandereycken W (2006a) Denial of illness in anorexia nervosa- a conceptual review: part 1 diagnostic significance and assessment. Eur Eat Disord Rev 14(5):341-351

Vandereycken W (2006b) Denial of illness in anorexia nervosa-a conceptual review: part 2 different forms and meanings. Eur Eat Disord Rev 14(5):352-368

Wakefield JC (1992) The concept of mental disorder. On the boundary between biological facts and social values. Am Psychol 47:373-388

Wakefield JC (1997) Diagnosing DSM-IV_-Part I: DSM-IV and the concept of disorder. Behav Res Ther 35(7):633-649

Wakefield JC (2013) DSM-5: an overview of changes and controversies. Clin Soc Work J 41(2):139-154

Wakefield JC (2016) Diagnostic issues and controversies in DSM-5: return of the false positives problem. Annu Rev Clin Psychol $12: 105-132$

Wheaton B (2007) The twain meet: distress, disorder and the continuing conundrum of categories. Health 11(3):303-19 (discussion 321-6)

Young SL (2011) Craving earth: understanding pica: the urge to eat clay, starch, ice, and chalk. Columbia University Press, New York

Young SL et al (2010) Association of pica with anemia and gastrointestinal distress among pregnant women in Zanzibar, Tanzania. Am J Trop Med Hyg 83(1):144-151

Zopf R, Contini E, Fowler C, Mondraty N, Williams MA (2016) Body distortions in anorexia nervosa: evidence for changed processing of multisensory bodily signals. Psychiatry Res 245:473-481

Publisher's Note Springer Nature remains neutral with regard to jurisdictional claims in published maps and institutional affiliations. 